\title{
One-step hydrothermal synthesis of 4-Metylimidazole Decorated Carbon Nanoparticles and Their Fluorescence Enhancement Sensing of Heavy Metal Ions
}

\author{
Tak H. Kim, ${ }^{1,2 *}$ Jung-ho Yun, ${ }^{3}$ Sue E. Boyd ${ }^{1}$ and Christopher L. Brown ${ }^{1}$ \\ ${ }^{1}$ School of Environment and Sciences, Griffith University, Nathan, QLD, 4111 Australia \\ ${ }^{2}$ Centre for Planetary Health and Food Security, Griffith University, Nathan, QLD, 4111 Australia \\ ${ }^{3}$ School of Chemical Engineering, The University of Queensland, St Lucia, QLD, 4067 Australia \\ *Corresponding Author: Tak H. Kim (t.kim@griffith.edu.au)
}

\begin{abstract}
We report fluorescent carbon nanoparticle decorated with 4-methylimidazole (4-MEI) prepared by a one-step hydrothermal synthesis. Upon binding to toxic heavy metals including $\mathrm{Hg}^{2+}$ and $\mathrm{Cd}^{2+}$, the fluorescence emission displayed notable enhancement. This is in contrast with the majority of other one-step synthesized fluorescent carbon nanoparticle sensing systems which exhibit quenching, due to the non-radiative energy transfer. In comparison, the decorated 4-MEI on the carbon nanoparticles prevents the non-radiative energy transfer when the analytes bind to the 4MEI. Further results on the fluorescence responses on the removal of the surface decorated 4-MEI and re-coordination of a reference 4-MEI against the same metal ions suggested that the 4-MEI on the carbon nanoparticles' surface plays a key role in the observed fluorescence enhancement. These features indicate an immense potential for further development of CNP based fluorescence enhancement sensing platforms for effective detection of analytes of interests.
\end{abstract}

Keywords: Carbon nanoparticle, fluorescence enhancement, fluorescence sensing, metal ion 


\section{Introduction}

Fluorescence material based sensing of metal ions offers numerous benefits over more traditional systems, such as detection sensitivity, ${ }^{1}$ fast response times ${ }^{2}$ and ease of measurement. ${ }^{3}$ The fluorescence sensing of metal ions typically relies on heavy atom effects in which complexation of heavy metal ions such as $\mathrm{Hg}(\mathrm{II}), \mathrm{Pb}(\mathrm{II})$ greatly reduces fluorescence intensity as compared to the free ligand or complexes of relatively light metal ions such as $\mathrm{Ca}(\mathrm{II})$ or $\mathrm{Zn}(\mathrm{II}){ }^{4,5}$ Due to high sensitivity and versatility, fluorescent organic dyes have been researched as fluorescent chemosensors for sensing of heavy metals.$^{6-9}$ However, chemosensory systems for heavy metal ion detection consist of combined fluorophores and macrocyclic molecules ${ }^{10,11}$ such as aza-crown ethers or sulfur containing receptors. ${ }^{7,12}$ Thus, the complexity of the synthesis and/or resulting low water solubility can be limiting factors. Likewise, recent progress in nanoparticle-based fluorescent probes such as those based on gold $^{13}$ and silver nanoparticles ${ }^{14}$ as well as systems based on semiconductor quantum $\operatorname{dots}^{15}$ have proved as effective heavy metal ion detectors. Unfortunately, they also suffer from complexity of synthesis and/or water solubility.

On the other hand, fluorescent carbon nanoparticles (CNPs) have exhibited considerable potential for a range of practical applications including biological applications, ${ }^{16,17}$ solid state lighting ${ }^{18}$ and sensing. ${ }^{19}$ For the last decade, one-step synthesized CNPs have been researched broadly due to their simplicity in preparation ${ }^{20}$, ease of dispersion in water ${ }^{21}$ and superior optical performance. ${ }^{22}$ The majority of the reported CNPs have been utilizing surface functionalization strategies such as decomposing carbon precursors containing other surface functionalization moieties in one pot. ${ }^{20}$ CNPs' sensing applications have especially been extensively researched due to the CNPs surface functionalities which play dual roles with the analytes. Firstly, the surface functionalities recognize the analytes by binding the analytes on the surface of the CNPs. Simultaneously, the analytes on 
the surface of the CNPs induce fluorescence quenching. Since the fluorescence of these CNPs depends on their surface states, the direct contact with these analytes onto the surface of the CNPs induces non-radiative energy and/or charge transfer, resulting in fluorescence quenching. Thus, CNP based sensors have been utilized for sensing of various materials such as heavy metal ions, ${ }^{23}$ anions $^{24}$ and a range of chemical systems. ${ }^{25}$

Herein, we report a fluorescent carbon nanoparticle-based sensing system, assisted by imidazole derived moieties derived from a one-step synthesis. Thus far, numerous CNP based sensors have been demonstrated based on their fluorescence quenching upon binding of analytes. The analyses of the multiple batches of the samples indicated that the decomposition of the carbon precursor (glucose) in the presence of a nitrogen source (ammonia) not only produce carbon nanoparticles but a heterocyclic compound, 4-methylimidazole (4-MEI). Although 4-MEI is typically produced from dicarbonyl, ammonia and aldehyde, it can be produced via degradation of glucose in aqueous ammonia solution, similar to Maillard reaction. ${ }^{26}$ This allows the formation of carbon nanoparticles and 4-MEI in one-step.

To probe the surface functionalities of the CNPs, the crude samples were fractionated, and their properties were carefully analyzed. The results indicated that most of the 4-MEI functionalities are not covalently tethered to the nanoparticles but coordinated to the nanoparticle surface. Since the majority of the functionalities are imidazole, the sensing of the metal ions showed a good sensitivity, similar to nitrogen containing fluorophore chemosensors, especially towards $\mathrm{Hg}^{2+}$ and $\mathrm{Cd}^{2+} \cdot{ }^{27}$ According to the previously reported CNP systems, our CNP based system would display quenching effects upon binding to analytes. Interestingly, however, the CNP samples showed fluorescence enhancement when they are introduced to analytes. Throughout the monitoring of the surface state changes upon binding, we found that the fluorescence enhancement is originated from 
changes in the CNPs' surface state due to the interactions between the 4-MEI and analytes. The imidazole moieties on the surface acts as metal ion recognition sites and are spaced away from the carbon cores due to their non-covalent binding motif. Therefore, direct contact with the analytes to the carbon core are inhibited whilst the energy transfer distances are increased upon the binding of the analytes. ${ }^{28,29}$ Results from our investigation on the filtration and re-coordination of the imidazole moieties onto the carbon cores suggested that the fluorescence of the CNPs and their responses toward the analytes also depends on the concentration of 4-MEI, as illustrated in Scheme

1. This represents a new strategy for designing CNP based fluorescence sensing platforms.

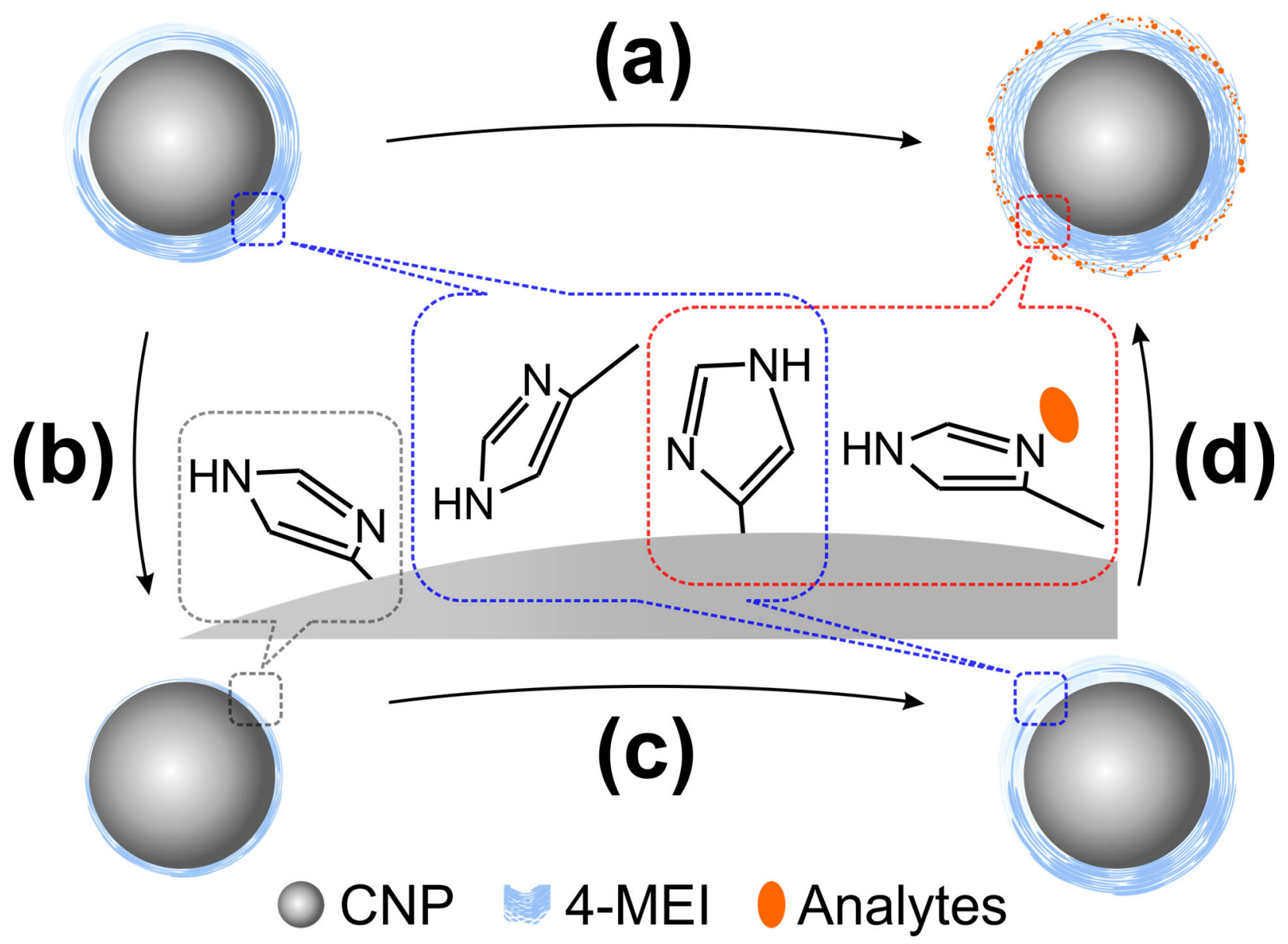

Scheme 1. Schematic representation of the two sensing systems studied in this work; (a) binding of the analytes onto the I-CNPs, (b) removal of the coordinated 4-MEI, resulting Filtered CNPs (F-CNPs), (c) re-coordination of 4-MEI, producing Re-coordinated CNPs (R-CNPs) and (d) binding of the analytes onto the R-CNPs. 


\section{Experimental}

\section{Carbon nanoparticle preparation}

I-CNPs were synthesized by reacting $1 \mathrm{M}$ glucose with $2 \mathrm{M}$ ammonium hydroxide in a hydrothermal reactor. Typically, $11 \mathrm{~mL}$ of $2 \mathrm{M}$ glucose solution $(2.7 \mathrm{~g}$ glucose, Chem-supply,

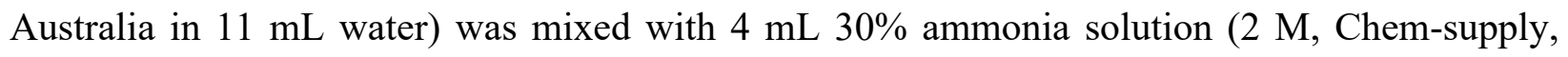
Australia) and stirred overnight. The mixture was transferred to a $30 \mathrm{~mL}$ PTFE lined hydrothermal reactor and the hydrothermal reactor was sealed and heated to $180{ }^{\circ} \mathrm{C}$ for 24 hours. The reaction mixture, containing the synthesized crude CNPs was mixed with water and then filtered through a $0.2 \mu \mathrm{m}$ nylon membrane in order to remove large aggregates. The water was isolated by freeze drying the crude CNPs. The crude CNPs were dissolved in a solvent mixture, consist of 4:1 tetrahydrofuran (Merck) and ethanol (Merck), and fractionated through a silica gel column (silica gel 60, Merck). Prior to the column fractionation, analytical thin layer chromatography was performed on a TLC plate (silica gel $60 \mathrm{~F}_{254}, 0.25 \mathrm{~mm}$ thick, Merck), three different fractions were observed by thin layer chromatography. The retention factors of the $1^{\text {st }}, 2^{\text {nd }}$ and $3^{\text {rd }}$ fractions were 0.84, 0.29 and 0 respectively. The fractionated CNPs were isolated from the solvent mixture by evaporation at reduced pressure. In order to investigate further on the role of 4-MEI and the nanoparticles, the 4-MEI in the $2^{\text {nd }}$ fraction samples (I-CNPs) were filtered through a gel filtration column (Sephadex G-10, Sigma-Aldrich) twice. The filtered CNPs (F-CNPs) were then freeze dried for further characterization and experiments. The Re-coordinated CNPs (R-CNPs) were prepared by stirring $10 \mathrm{~mL}$ of the F-CNPs (UV-vis absorbance 0.18 at $360 \mathrm{~nm}$ ) solution with 10 $\mathrm{mL}$ of 4-MEI (Sigma-Aldrich) solutions at $60^{\circ} \mathrm{C}$ for 24 hours under reflux. The mixtures were cooled to room temperature and stirred for 24 hours further. Blank CNPs (B-CNPs) were prepared 
by using $11 \mathrm{~mL}$ of $1 \mathrm{M}$ glucose solution and $4 \mathrm{~mL}$ of water was added instead of ammonia solution. The conditions of the synthesis were kept identical to the preparation of I-CNPs. The assynthesized B-CNPs were filtered through a $0.2 \mu \mathrm{m}$ nylon membrane and freeze dried to isolate from the water.

\section{Spectroscopic materials and methods}

Deionized water $(18 \mathrm{M} \Omega \cdot \mathrm{cm})$ was used to prepare all aqueous solutions including metal ion and the MI/CNP solutions. The MI/CNP solutions for the fluorescence tests were prepared at $5 \mu \mathrm{g} \cdot \mathrm{mL}^{-}$

${ }^{1}$ (UV-vis absorbance 0.09 at $360 \mathrm{~nm}$ ). Metal ion solutions were prepared by dissolving chloride metal salts (Chem-supply, Australia) in water. Samples for ${ }^{1}$ HNMR spectra of the CNPs with the metal ions $\left(\mathrm{Cd}^{2+}\right.$ and $\left.\mathrm{Hg}^{2+}\right)$ were prepared by dissolving the CNPs in $d_{6}$-DMSO followed by mixing with cadmium chloride or mercury nitrate (Chem-supply, Australia). The samples for the fluorescence was prepared by mixing $1 \mathrm{~mL}$ of the $\mathrm{CNP}$ solution and $1 \mathrm{~mL}$ of the metal ion solution. Prior to the test, all other CNPs were further diluted by adjusting UV-vis absorbance to 0.09 at 360 $\mathrm{nm}$. The 4-MEI aqueous solutions for metal ion titrations were also prepared by adjusting UV-vis absorbance to 0.09 at $360 \mathrm{~nm}$ and the molarity was $50 \mathrm{mM}$. The fluorescence of the mixtures was measured as soon as the sample solutions and the analytes were mixed. All optical properties, reported herein, were measured at $\mathrm{pH} 7$.

\section{Characterizations}

Atomic force microscopy (AFM) images were captured on an NT-MDT NTEGRA Spectra AFM using a semi-contact mode. Transmission electron microscopy (TEM) images were captured on a JEOL JEM-1010 and high resolution TEM images were captured on a Hitachi HT7700. X-ray photoelectron spectroscopy (XPS) spectra were acquired using a Kratos Axis ULTRA X-ray photoelectron spectrometer incorporating a $165 \mathrm{~mm}$ hemispherical electron energy analyzer. 
Calculations of atomic concentrations and peak fittings of the high-resolution data were carried out using the CasaXPS software package (v2.2.73). Fourier transform infrared (FTIR) spectra were recorded on a PerkinElmer Spectrum Two IR spectrometer equipped with a diamond attenuated total reflectance (ATR) attachment. ATR corrections were applied using PerkinElmer Spectrum software package (v10.03.09) after acquisitions. Nuclear magnetic resonance (NMR) spectra were acquired on a Varian $400 \mathrm{MHz}$ Unity INOVA spectrometer in $d_{6}$-DMSO at the temperatures quoted. ${ }^{1} \mathrm{H}$ NMR chemical shifts are referenced to solvent residuals, taken as 2.49 ( $d_{6}$-DMSO). All NMR spectra were processed using the MestReNova software package (v11.0.4). UV-visible (UVvis) absorption spectra for dispersed samples were measured on an Agilent 8453 UV-vis spectrometer. Fluorescence spectra were measured by a Thermo Lumina fluorescence spectrometer. Fluorescence decays were recorded by a using $374 \mathrm{~nm}$ picosecond pulse laser excitation source in an Edinburgh Photonics FLS920 photoluminescence spectrometer. Fluorescence lifetimes were estimated from the measured fluorescence decay curves and the laser profiles using nonlinear least-square iterative fittings on F900 software package (v7.2.1). Quantum yields were measured by comparing quantum yield standards at $360 \mathrm{~nm}$ excitation (quinine sulfate, 54\%). All samples for quantum yield were prepared by diluting the original samples until their absorbance was 0.045 for the excitation wavelength.

\section{Results and discussions}

Imidazole decorated CNPs (I-CNPs) were synthesized by decomposing $1 \mathrm{M}$ glucose with $2 \mathrm{M}$ ammonium hydroxide hydrothermally at $180^{\circ} \mathrm{C}$ for 24 hours. The evaporation of the water from the crude reaction mixture resulted in a caramel like crude product which is consistent with a previously reported system. ${ }^{26}$ The crude product was column fractionated (see experimental) in order to obtain nanoparticles containing 4-MEI. Since we observed that the nanoparticles from the 
$2^{\text {nd }}$ fraction contains 4-methylimidazole, our discussion is focused on the $2^{\text {nd }}$ fraction (I-CNPs). However, further information on the other fraction is available in Supplementary Information.
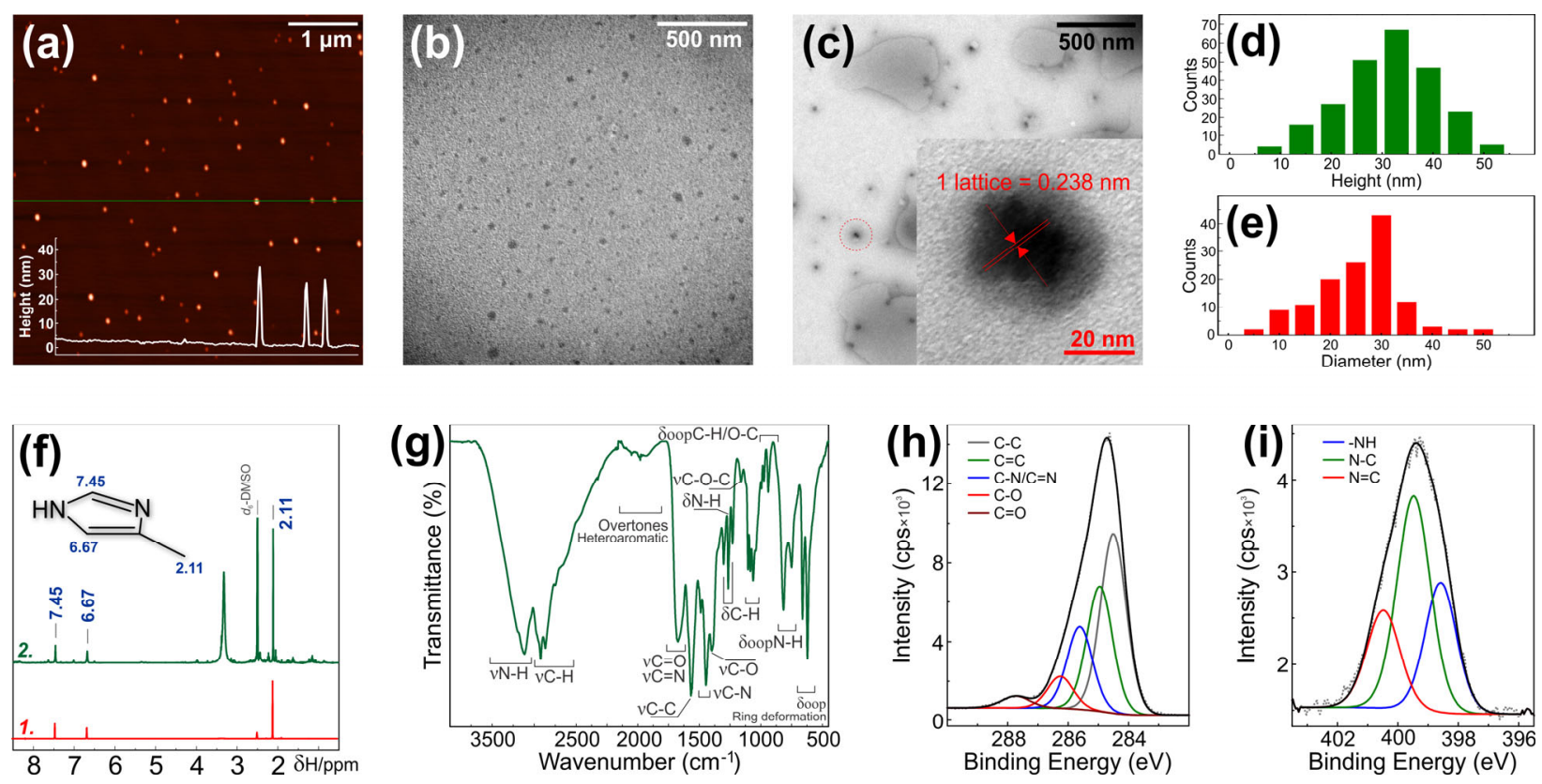

Figure 1. (a) AFM and (b) TEM image obtained from the $2^{\text {nd }}$ fraction sample (I-CNPs), (c) HRTEM images of I-CNPs (inset: high resolution image of the particle in red circle), particle size distributions obtained from (d) AFM and (e) TEM image, (f) ${ }^{1}$ HNMR spectrum of 1. 4-MEI vs. 2. I-CNPs (400 MHz, 296K, $d_{6}$-DMSO), (g) IR, XPS high resolution (h) C1s and (i) N1s spectrum of I-CNPs.

\section{Physiochemical properties}

As presented in Figure 1, the particles in I-CNPs were clearly observable from both atomic force microscopy (AFM) and transmission electron microscopy (TEM) images. The particle sizes, analyzed from the AFM and TEM, were comparable each other with the majority of the particles are between 15 to $35 \mathrm{~nm}$. High resolution TEM (HRTEM) characterization in Figure 1 (c) suggests that the carbon core structure is crystal-like. Successive planes of carbon lattices are observed with an interplanar distance of $\sim 0.24 \mathrm{~nm}$. The AFM analyses of the crude sample indicated that it not only contained nanoparticles, but also large sheet-like materials (Figure S1). However, the particle 
size was identical to the size of the I-CNPs. The particle size of the $1^{\text {st }}$ fraction was also identical to the particle sizes observed from the crude CNPs and I-CNPs ( $2^{\text {nd }}$ fraction). No large sheet-like materials were found in both $1^{\text {st }}$ and $2^{\text {nd }}$ fractions, indicating the sheet-like materials reside in the immobile $3^{\text {rd }}$ fraction.

${ }^{1} \mathrm{H}$ NMR characterizations indicated that the crude samples and I-CNPs contain 4-MEI. As presented in Figure 1 (f), the chemical shifts observed from ${ }^{1} \mathrm{H}$ NMR characterizations were consistent with the reference 4-MEI (Comparison of the ${ }^{1} \mathrm{H}$ NMR spectra from the crude, $1^{\text {st }}$ and $2^{\text {nd }}$ fraction samples are available in Supplementary Information).

The IR spectrum obtained for the I-CNPs are presented in Figure $1(\mathrm{~g})$. The spectrum exhibit N-H stretches between 3200 and $3000 \mathrm{~cm}^{-1}$. The additional bands at $1446 \mathrm{~cm}^{-1}$ and $1265 \mathrm{~cm}^{-1}$ which correspond to $\mathrm{C}-\mathrm{N}$ stretch along with $\mathrm{N}-\mathrm{H}$ bend, supporting the presence of amine like functionalities in the I-CNPs. Furthermore, overtones from hetero-aromatics were observed between 2100 and $1900 \mathrm{~cm}^{-1}$ which further supports the presence of the imidazole moieties in the sample. It is notable that these distinctive peaks were in line with the IR spectrum obtained from the reference 4-MEI (Figure S3) and the observations made in the ${ }^{1} \mathrm{H}$ NMR spectroscopy studies. However, these bands are not observable from the $1^{\text {st }}$ fraction sample.

It is also notable that $\mathrm{C}=\mathrm{O}$ stretch at $1680 \mathrm{~cm}^{-1}$ and $\mathrm{C}-\mathrm{O}$ stretch at $1403 \mathrm{~cm}^{-1}$ were observable from both $1^{\text {st }}$ fraction and I-CNPs, indicating the existence of the structural defects and/or oxidized products on the surface of the particles. In an effort to obtain a better understanding of the 4-MEI on the surface of I-CNPs, 4-MEI Filtered CNPs (F-CNPs, filtered through a column containing Sephadex G-10) and Blank CNPs (B-CNPs, sample prepared without ammonia) were prepared and examined. The IR spectrum of the F-CNPs was relatively similar to the IR spectrum of the $1^{\text {st }}$ 
fraction and B-CNPs, indicating the majority of the 4-MEI exist in the I-CNPs was not covalently tethered to the surface of the nanoparticles. The $\mathrm{C}=\mathrm{O}$ and $\mathrm{C}-\mathrm{O}$ stretches are evident across the samples examined indicating the oxygen containing surface functionalities are available regardless the 4-MEI or ammonia.

X-ray photoelectron spectroscopy (XPS) survey spectrum of I-CNPs indicates that the majority of the sample consist of carbon (78\%, Figure S7). The high resolution C1s spectrum in Figure 1 (h) was deconvoluted into five peaks which corresponds to $\mathrm{sp}^{3} \mathrm{C}-\mathrm{C}$ at $284.5 \mathrm{eV}, \mathrm{sp}^{2} \mathrm{C}=\mathrm{C}$ at $284.9 \mathrm{eV}$, $\mathrm{C}-\mathrm{N} / \mathrm{C}=\mathrm{N}$ at $285.6 \mathrm{eV}, \mathrm{C}-\mathrm{O}$ at $286.3 \mathrm{eV}$, and $\mathrm{C}=\mathrm{O}$ at $287.7 \mathrm{eV}$, respectively. The deconvoluted peaks of $\mathrm{C}-\mathrm{O}$ and $\mathrm{C}=\mathrm{O}$ further confirms the existence of oxidized surface functionalities. The highresolution N 1s spectrum of I-CNPs could be deconvoluted into three peaks, which corresponds to $-\mathrm{NH}$ at $398.6 \mathrm{eV}, \mathrm{N}-\mathrm{C}$ at $399.5 \mathrm{eV}$ and $\mathrm{N}=\mathrm{C}$ at $400.5 \mathrm{eV}$. The distinct $\mathrm{N}-\mathrm{C}$ and $\mathrm{N}=\mathrm{C}$ is likely to have originated from imidazole.

\section{Optical properties}

The UV-vis absorption of the I-CNPs in Figure 4 (a) displayed a distinctive peak at $275 \mathrm{~nm}$ with a broad shoulder peak spanning from $290 \mathrm{~nm}$ to $325 \mathrm{~nm}$. These absorption characteristics could be observed in the crude sample (Figure S8 (a)). Notably, the absorbance was much prominent in the I-CNPs at the same CNP concentrations $\left(5 \mu \mathrm{g} \cdot \mathrm{mL}^{-1}\right)$. A broad absorption peak between 270 and $290 \mathrm{~nm}$ from the $1^{\text {st }}$ fraction was observed, in contrast to the distinctive peak from the I-CNPs. This may indicate the absorption peak and/or the shoulder, at 275 and $295 \mathrm{~nm}$ respectively, are related to the imidazole. To elucidate these observations, UV-vis absorption of 4-MEI was measured (Figure S9 (a)). The UV-vis absorption spectrum of 4-MEI displayed a peak at $295 \mathrm{~nm}$ which is assignable to $\pi-\pi^{*}$ transition of $\mathrm{C}=\mathrm{N}$ on the 4-MEI ring. ${ }^{30}$ This further indicates the shoulder peak from the I-CNPs corresponds to the imidazole moieties exist on the I-CNPs. 

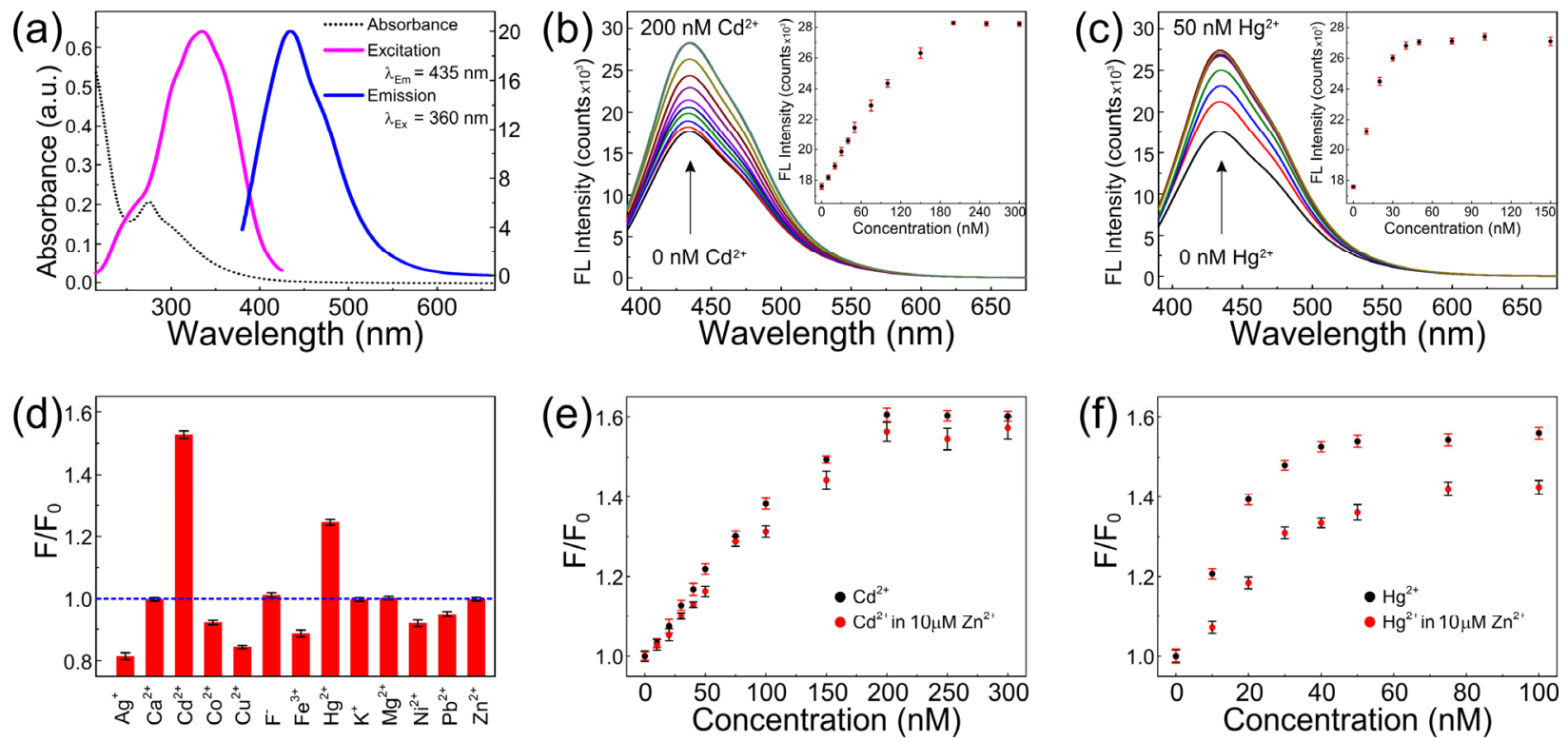

Figure 2. (a) UV-vis, excitation and emission spectrum of the I-CNPs, fluorescence spectra responses of the I-CNPs on the addition of (b) $\mathrm{Cd} 2+$ and (c) $\mathrm{Hg} 2+$ ion (Inset: fluorescence intensity measured at $435 \mathrm{~nm}$ ), changes in the fluorescence intensities, in the presence of the excessive competing ion $\left(\mathrm{Zn}^{2+}, 10 \mu \mathrm{M}\right)$, on the addition of (e) $\mathrm{Cd}^{2+}$ and (f) $\mathrm{Hg}^{2+}$ ion and (d) fluorescence responses of the I-CNPs on the addition of various metal ions at $5 \mu \mathrm{M}$ concentration (blue horizontal line represents the reference relative intensity).

The fluorescent excitation spectrum of the I-CNPs at $\lambda_{\mathrm{Em}}=435 \mathrm{~nm}$ in Figure 4 (a) showed a symmetric peak between 310 and $365 \mathrm{~nm}$ which parallels to the UV-vis absorption range of the shoulder peak from the UV-vis spectrum. A notable shoulder in the excitation spectrum at $275 \mathrm{~nm}$ is in line with the distinctive UV-vis peak accordingly. To obtain further insights on the functional groups on the CNPs including the crude and $1^{\text {st }}$ fraction samples, fluorescence of the sample CNPs were measured at $\lambda_{\mathrm{Ext}}=265,275$ and $285 \mathrm{~nm}$ (Figure S8). Both the crude CNPs and the $1^{\text {st }}$ fraction sample displayed hypsochromic shifts whilst the emission spectra from the I-CNPs were excitation independent (emission peak at $385 \mathrm{~nm}$ ). The observation from the I-CNPs agrees with Kasha's rule $^{31}$ thus, the fluorescence of the I-CNPs at $\lambda_{\mathrm{Ext}}=\sim 275 \mathrm{~nm}$ is originated from the imidazole. 
Fluorescent emission at $\lambda_{\mathrm{Ex}}=360 \mathrm{~nm}$ in Figure 2 (a) displayed a blue emission with an emission peak at $435 \mathrm{~nm}$. Fluorescence spectra of the samples at excitation wavelengths from 300 to 400 $\mathrm{nm}$ displayed excitation dependency on their fluorescence emission (Figure S8), similar to the previously reported systems. ${ }^{24,32}$ This also correlates well with the broad UV-vis absorption shoulder from 300 to $400 \mathrm{~nm}$ in the UV-vis spectra which is originated from the carbon nanoparticle cores rather than their functional groups.

\section{Metal ion sensing}

The addition of metal ions to the I-CNPs led to various responses, based on the metal ions tested. Figure 2 (d) displays the fluorescence responses of the I-CNPs after the addition of several chloride salts of metal ions. Upon the addition of an equivalent amount of $5 \mu \mathrm{M}$ the metal ions to aqueous solutions of the I-CNPs, only $\mathrm{Cd}^{2+}$ and $\mathrm{Hg}^{2+}$ ions enhance the fluorescence signal, while the addition of $\mathrm{Ag}^{+}, \mathrm{Co}^{2+}, \mathrm{Fe}^{3+}, \mathrm{Ni}^{2+}$ and $\mathrm{Pb}^{2+}$ ions causes fluorescence quenching. No significant fluorescence signal changes were noted from the other cations/anions. The fluorescence spectra for titrations of the I-CNPs with $\mathrm{Cd}^{2+}$ and $\mathrm{Hg}^{2+}$ are shown in Figure 2 (b) and (c). The fluorescence signals started to increase upon the addition of $\mathrm{Cd}^{2+}$ and $\mathrm{Hg}^{2+}$. The FL intensity was increased up to $62 \%$ and $56 \%$ for $\mathrm{Cd}^{2+}$ and $\mathrm{Hg}^{2+}$ at $200 \mathrm{nM}$ and $50 \mathrm{nM}$ metal ion concentration respectively. The intensity increased linearly with the concentration of $\mathrm{Cd}^{2+}$ from 0 to $100 \mathrm{nM}$ with linearity dependent coefficient, $R^{2}=0.997$ whilst $\mathrm{Hg}^{2+}$ showed linear responses from 0 to $40 \mathrm{nM}$ with $R^{2}=$ 0.985 . The intensity reached a plateau at $250 \mathrm{nM} \mathrm{Cd}^{2+}$ and $100 \mathrm{nM} \mathrm{Hg}^{2+}$, indicating the complete complexation at their maximum intensity increases. On the basis of the fluorescence titrations of the I-CNPs, we have determined stability constants, $K a$ for $\mathrm{Cd}^{2+}$ to be $3.97 \mathrm{M}^{-1}$ and $\mathrm{Hg}^{2+}$ to be 20.0 $\mathrm{M}^{-1}$ (See Supplementary Information for further details). ${ }^{33,34}$ No spectral shifts in the presence of the metal ions were noted, in contrast to molecular based metal ion sensing systems reported 
previously. ${ }^{10}$ Competition experiments in which the I-CNPs was exposed to excess $\mathrm{Zn}^{2+}$, then to the addition of $\mathrm{Cd}^{2+}$ and $\mathrm{Hg}^{2+}$ in Figure 2 (e) and (f), lead to 2 to $10 \%$ reduction in the emission intensity, in the order $\mathrm{Cd}^{2+}$ and $\mathrm{Hg}^{2+}$. It is also noticeable that the reduction depended on the metal ion concentrations. This observation is consistent with the relative affinities that would be anticipated on the examination of the fluorescence without $\mathrm{Zn}^{2+}$.

The aqueous 4-MEI (50 $\mu \mathrm{M}), \mathrm{F}-\mathrm{CNP}$ and B-CNP solutions were titrated with $\mathrm{Cd}^{2+}$ and $\mathrm{Hg}^{2+}$ under the identical conditions to attain a better comparison. Unlike to the result from the I-CNPs, no significant fluorescence changes were observed across the control samples examined (Figure S11). These results indicate that the 4-MEI on I-CNPs may associate with metal ions and the carbon nanoparticles by cooperative binding, similar to nitrogen containing fluorescence sensing molecules. ${ }^{35,36}$ It is also worth mentioning that the fluorescence intensities of F-CNPs were notably quenched, in comparison with I-CNPs, which is consistent with the observations reported previously. ${ }^{37}$ This further confirms that the direct interaction with the analytes and CNP cores results in the quenching of the fluorescence. 

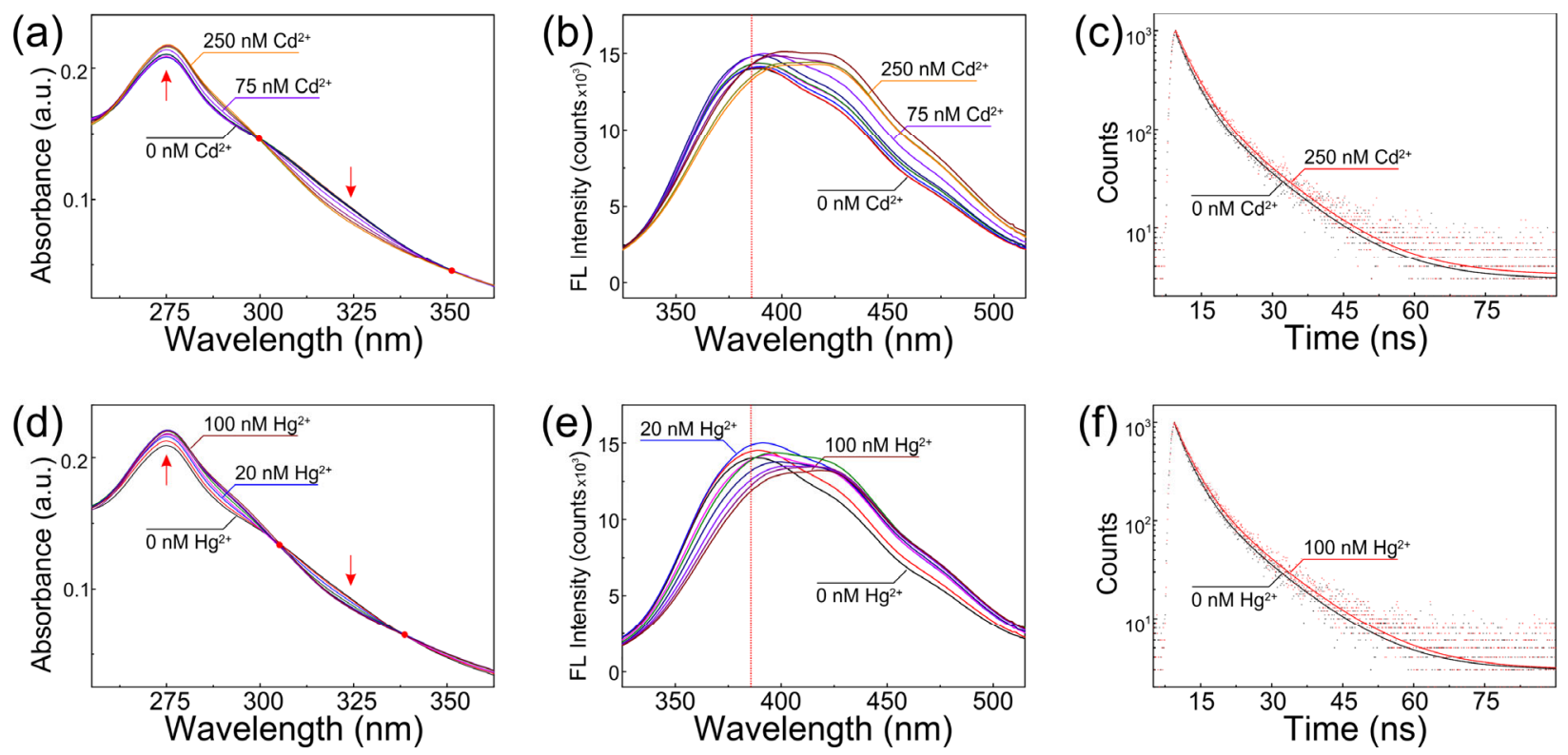

Figure 3. Changes in (a), (d) UV-vis, (b), (e) fluorescence spectra at $275 \mathrm{~nm}$ excitation and (c), (f) fluorescence decay curves and fittings of the I-CNPs on the addition of $\mathrm{Cd}^{2+}$ and $\mathrm{Hg}^{2+}$ ion.

To probe the association of the metal ions and CNPs, UV-vis spectra of the titrations of the I-CNPs with $\mathrm{Cd}^{2+}$ and $\mathrm{Hg}^{2+}$ were examined. As presented in Figure $3(\mathrm{a})$ and (d). Only small changes $\left(\Delta_{\text {abs }}\right.$ $=0.002$ at $360 \mathrm{~nm}$ ) in the absorption occurred during the titrations, indicating that the fluorescence emission intensity changes are predominantly related to the changes in quantum yield. ${ }^{38}$ The absorption spectra from both $\mathrm{Cd}^{2+}$ and $\mathrm{Hg}^{2+}$ displayed two isosbestic points, supporting the complexation of the metal ions and changes in the particles' surface states upon binding. ${ }^{39-42}$ Notably, the UV-vis absorption at $275 \mathrm{~nm}$ and $290 \mathrm{~nm}$ region showed increase while the absorption near $325 \mathrm{~nm}$ showed decrease. The changes in the absorptions are ascribable to the changes to the imidazole functionalities on the surface since these peaks are comparable to the peaks observed from the UV-vis characterizations in Figure 2 (a). Consequently, the absorption changes upon the addition of $\mathrm{Cd}^{2+}$ and $\mathrm{Hg}^{2+}$ support the changes of the subsequent surface states which may rigidify the 4-MEI surface functionalities and prevent nonradiative decay subsequently. ${ }^{42,43}$ The UV-vis 
absorption displayed minimal changes over $200 \mathrm{nM} \mathrm{Cd}^{2+}$ and $50 \mathrm{nM} \mathrm{Hg}^{2+}$ concentrations supporting the I-CNPs complete complexation with the analytes.

Control UV-vis titration experiments using 4-MEI solution and F-CNPs were conducted. The UVvis of the titrations of the 4-MEI solution with $\mathrm{Cd}^{2+}$ and $\mathrm{Hg}^{2+}$ displayed very slight changes in their absorption $\left(\Delta_{\mathrm{abs}}=0.004\right.$ at $275 \mathrm{~nm}$, Figure S12) which are consistent with the slight changes observed in the fluorescence. Nevertheless, the UV-vis absorption changes at 275 and $325 \mathrm{~nm}$ regions with one isosbestic point confirm the complexation of the metal ion with 4-MEI which may be attributed to the energy transitions between the metal ions and the 4-MEI upon the coordination. ${ }^{42}$ The UV-vis responses from the F-CNPs showed insignificant changes ( $\Delta_{\mathrm{abs}}=0.008$ at $275 \mathrm{~nm}$ ) which also showed similar fluorescence responses with the 4-MEI solutions. However, unlike the 4-MEI, no isosbestic points were noted suggesting no significant interaction between the F-CNPs and the metal ions tested in the absence of the 4-MEI, in contrast with the cases of ICNPs.

As displayed in Figure 3 (b) and (e), the fluorescence spectra taken over the course of the same titrations of the I-CNPs with $\mathrm{Cd}^{2+}$ and $\mathrm{Hg}^{2+}$ at $\lambda_{\mathrm{Ext}}=275 \mathrm{~nm}$ were notably different from the fluorescence spectra measured at $\lambda_{\text {Ext }}=360 \mathrm{~nm}$ (vs. Figure 2 (b) and (c)). It was expected to see obvious fluorescence enhancement at $275 \mathrm{~nm}$ excitation. However, a slight fluorescence enhancement was observed at lower metal ion concentrations followed by hypochromic effects with bathochromic shifts. The fluorescence decays were measured at the samples' complete complexation and compared with blank samples (Figure $3(\mathrm{c})$ and (f), $\lambda_{\mathrm{Ex}}=374 \mathrm{~nm}$ ). The decay curves were bested fitted by a bi-exponential function, indicating two different fluorescence spices exist in the I-CNPs. The comparison with the reference decay indicates small but notable changes in fluorescence spices exist in the I-CNPs. The ratios between $\tau_{1}$ and $\tau_{2}$ were comparably similar 
throughout the measurement (Table S1). At the samples' complete complexation, the decay fitting curves showed FL decay increase for both $\mathrm{Cd}^{2+}$ and $\mathrm{Hg}^{2+}$, indicating the excited states of the ICNPs were extended by the coordinated metal ions onto the 4-MEI.

In order to further examine the coordination of the metal ions, ${ }^{1} \mathrm{HNMR}$ spectra of the I-CNPs doped with $\mathrm{Cd}^{2+}$ and $\mathrm{Hg}^{2+}$ were acquired. As presented Figure 4, chemical shifts of the 4-MEI due to the addition of $\mathrm{Cd}^{2+}$ and $\mathrm{Hg}^{2+}$ was evident. Chemical shifts increase with respect to the nonprotonated species when the pair of electrons in the nitrogen heterocycle are protonated. ${ }^{44}$ On the other hand, a decrease in the electron density of the imidazole yields downfield shifts at all ring positions which is inversely proportional to distance.

The observations from the chemical shifts are well aligned with the optical property changes observed in the fluorescence spectral shifts measured at $\lambda_{\mathrm{Ext}}=275 \mathrm{~nm}$ (Figure 3 (b) and (e)) and deviations on the decay responses (Figure 3 (d) and (f)). This also confirms the changes of the 4MEI on the surface of the carbon cores when metal ions are coordinated. The bindings of the metal to the 4-MEI would result in energy level changes followed by the CNPs' surface functional group changes (rigidification?). ${ }^{42}$ The changes of the 4-MEI may result in the increased FL emission intensity via radiative energy transfer from the CNPs to the 4-MEI and the coordinated metal. ${ }^{42,43}$ 


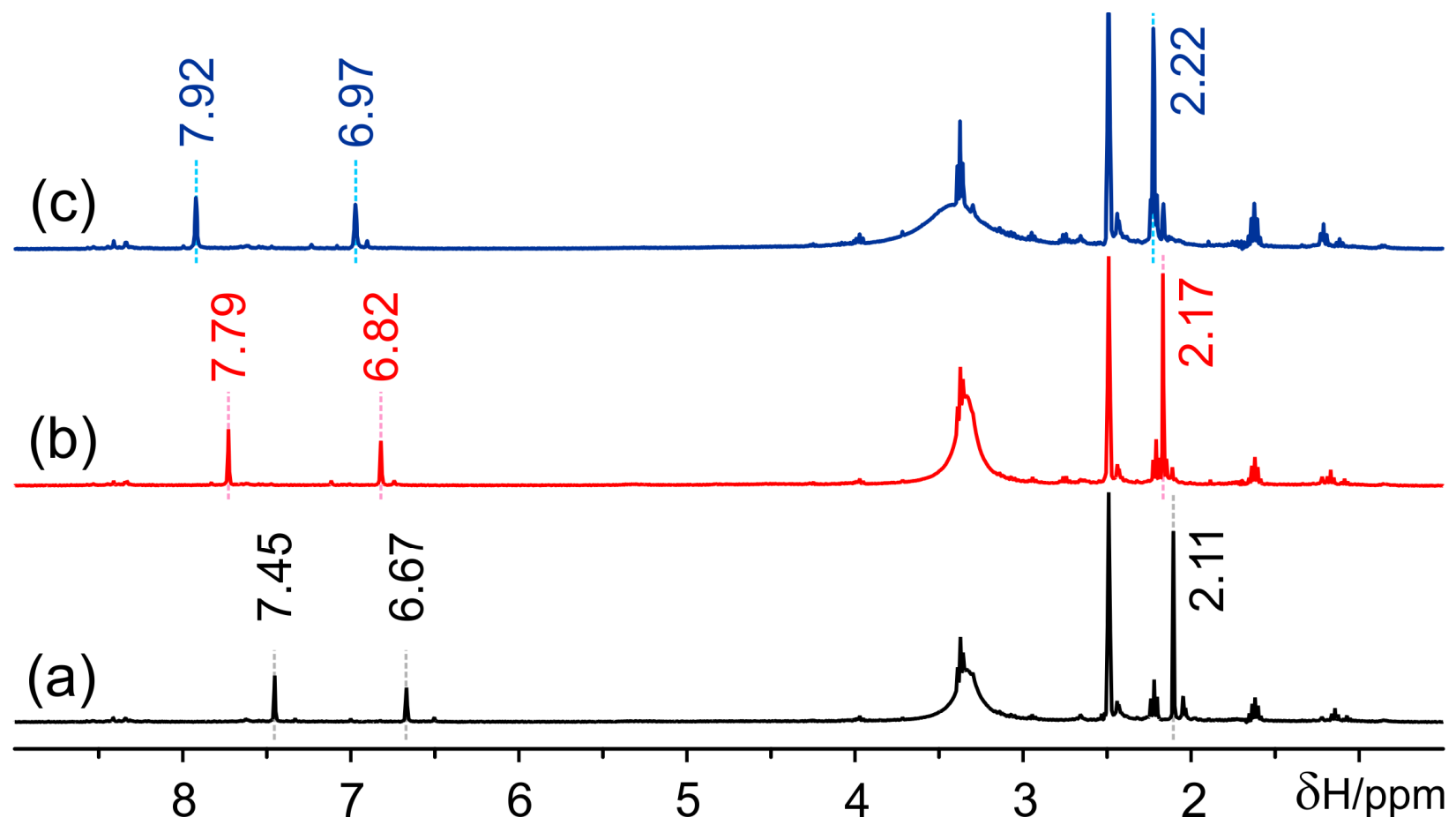

Figure 4. ${ }^{1} \mathrm{HNMR}$ Spectral shifts (400 MHz, 296K, $d_{6}$-DMSO) of (a) I-CNPs in comparison with the addition of (b) $\mathrm{Cd}^{2+}$ and (c) $\mathrm{Hg}^{2+}$ ion. (Note: ${ }^{199} \mathrm{Hg} \mathrm{NMR}$ experiments were attempted. However, due to the solubility of mercury nitrate, no meaningful results were obtained) 

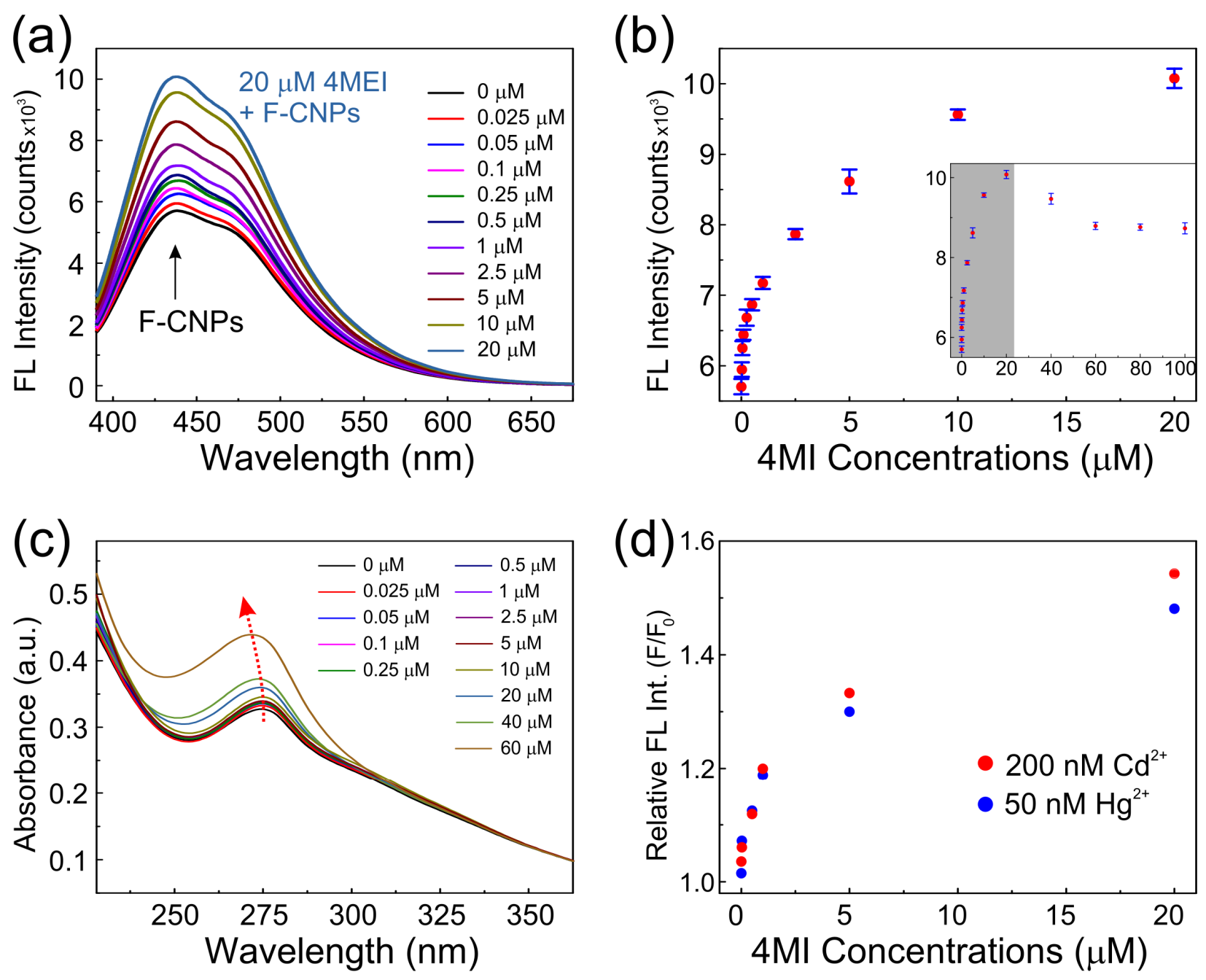

Figure 5. (a) Fluorescence spectra of the F-CNPs on the addition of 4-MEI solution, (b) expansion of the fluorescence intensity measured at $435 \mathrm{~nm}$ in Inset (grey shaded area), (c) UV-vis spectra and (d) fluorescence responses of the R-CNPs (F-CNPs re-coordinated with $20 \mu \mathrm{M} 4$-MEI solution) on the addition of $\mathrm{Cd}^{2+}$ and $\mathrm{Hg}^{2+}$ ions (full titration data is presented in Figure $\mathrm{S} 13$ ).

Re-coordination of 4-MEI and metal ion responses

It is of interest to establish the extent to which 4-MEI coordinates the surface of the CNPs and the changes in the fluorescence. Hence, the F-CNPs were re-coordinated with 4-MEI solutions from $0.025 \mu$ to $60 \mu \mathrm{M}$ at $60^{\circ} \mathrm{C}$ for 24 hours, producing re-coordinated CNPs (R-CNPs). As displayed in Figure 4 (a) and (b), the fluorescence of the resulting R-CNPs showed notable enhancement. The fluorescence reached its maximum at $20 \mu \mathrm{M}$ 4-MEI followed by a slight hypochromic effect 
at concentrations higher than $40 \mu \mathrm{M}$, indicating a complete coordination at $20 \mu \mathrm{M} 4-\mathrm{MEI}$. Stability constant $K_{a}$ of R-CNPs was determined at $0.85 \mathrm{M}^{-1}$, based on the titrations of the R-CNPs and their fluorescence responses. ${ }^{11,33}$ Similar to I-CNPs, the fluorescence emissions at $\lambda_{\text {Ext }}=\sim 275 \mathrm{~nm}$ showed no spectral shifts, indicating the re-coordination of the 4-MEI onto the CNPs' surface (Figure S9 (f)). The UV-vis spectra obtained from the R-CNPs (Figure 4 (c)) displayed an isosbestic point at $235 \mathrm{~nm}$ with a hyperchromic effect at $275 \mathrm{~nm}$, confirming the re-coordination of the 4-MEI onto the CNPs' surface. Hypsochromic shifts and disappearance of the isosbestic point over $40 \mu \mathrm{M}$ 4-MEI reflected that the coordinating abilities and the stabilities of the F-CNPs reached its' equilibrium at $20 \mu \mathrm{M} .{ }^{45}$ These observations from the UV-vis correlate well with the fluorescence responses over $40 \mu \mathrm{M}$ 4-MEI in Figure 4 (b). Titrations of the R-CNPs with $\mathrm{Cd}^{2+}$ and $\mathrm{Hg}^{2+}$ are presented in Figure 4 (d). The responses upon the addition of the obtained were also associate well with the 4-MEI concentrations and the responses of the $20 \mu \mathrm{M} 4$-MEI recoordinated R-CNPs were similar to I-CNPs responses in Figure 2 (e) and (f).

\section{Conclusions}

We have demonstrated a sensitive and selective fluorescence enhancement CNP sensing platform using a one-step hydrothermal synthetic strategy. In contrast to the majority of one-step CNP systems, the fluorescence intensity showed enhancement upon the bindings to the analytes. The changes of the CNPs optical properties with the analytes indicated that the 4-MEI functionality is coordinated to the CNP preventing direct interactions with the analytes and CNP cores. On the other hand, CNPs without the 4-MEI functionality showed notable quenching when the analytes were introduced. Re-coordination of 4-MEI to the bare CNPs further enabled the fluorescence enhancement when the analytes were introduced, confirming the coordination of the 4-MEI on the 
CNPs surface. Our study provided a simple but effective strategy for CNP fluorescence sensing platform.

\section{Conflicts of Interest}

Authors declare no conflicts of interest

\section{Supplementary Information}

Supplementary information is available

\section{Acknowledgement}

Authors acknowledge Dr Barry Wood, the Centre for Microscopy and Microanalysis for his assistance on the XPS analysis and suggestions. T.K thanks Mr Richard Webb the Centre for Microscopy and Microanalysis for his assistance on the TEM characterization. 


\section{References}

(1) Carter, K. P.; Young, A. M.; Palmer, A. E. Fluorescent Sensors for Measuring Metal Ions in Living Systems. Chem. Rev. 2014, 114 (8), 4564-4601.

https://doi.org/10.1021/cr400546e.

(2) Choudhury, S. D.; Mohanty, J.; Pal, H.; Bhasikuttan, A. C. Cooperative Metal Ion Binding to a Cucurbit[7]Uril - Thioflavin T Complex: Demonstration of a Stimulus-Responsive Fluorescent Supramolecular Capsule. J. Am. Chem. Soc. 2010, 132 (4), 1395-1401. https://doi.org/10.1021/ja908795y.

(3) Bian, R.-X.; Wu, X.-T.; Chai, F.; Li, L.; Zhang, L.-Y.; Wang, T.-T.; Wang, C.-G.; Su, Z.M. Facile Preparation of Fluorescent Au Nanoclusters-Based Test Papers for Recyclable Detection of $\mathrm{Hg} 2+$ and Pb2+. Sensors Actuators B Chem. 2017, 241, 592-600. https://doi.org/10.1016/j.snb.2016.10.120.

(4) Silva, A. P.; Gunaratne, H. Q. N.; Gunnlaugsson, T.; Huxley, A. J. M.; McCoy, C. P.; Rademacher, J. T.; Rice, T. E. Signaling Recognition Events with Fluorescent Sensors and Switches. Chem. Rev. 1997, 97 (5), 1515-1566. https://doi.org/10.1021/cr960386p.

(5) Hancock, R. D. The Pyridyl Group in Ligand Design for Selective Metal Ion Complexation and Sensing. Chem. Soc. Rev. 2013, 42 (4), 1500-1524. https://doi.org/10.1039/C2CS35224A.

(6) Guo, X.; Qian, X.; Jia, L. A Highly Selective and Sensitive Fluorescent Chemosensor for Hg2+ in Neutral Buffer Aqueous Solution. J. Am. Chem. Soc. 2004, 126 (8), 2272-2273. https://doi.org/10.1021/ja037604y.

(7) Yoon, S.; Miller, E. W.; He, Q.; Do, P. H.; Chang, C. J. A Bright and Specific Fluorescent Sensor for Mercury in Water, Cells, and Tissue. Angew. Chemie - Int. Ed. 2007, 46 (35), 6658-6661. https://doi.org/10.1002/anie.200701785.

(8) W. Nugent, J.; Lee, H.; Lee, H.-S.; H. Reibenspies, J.; D. Hancock, R. Mechanism of Chelation Enhanced Fluorescence in Complexes of Cadmium(Ii), and a Possible New Type of Anion Sensor. Chem. Commun. 2013, 49 (84), 9749-9751. https://doi.org/10.1039/b000000x.

(9) Li, J.; Wu, Y.; Song, F.; Wei, G.; Cheng, Y.; Zhu, C. A Highly Selective and Sensitive Polymer-Based OFF-ON Fluorescent Sensor for Hg2+ Detection Incorporating Salen and Perylenyl Moieties. J. Mater. Chem. 2012, 22 (2), 478. https://doi.org/10.1039/c1jm14037j.

(10) McFarland, S. A.; Finney, N. S. Fluorescent Chemosensors Based on Conformational Restriction of a Biaryl Fluorophore [16]. Journal of the American Chemical Society. American Chemical Society February 2001, pp 1260-1261. https://doi.org/10.1021/ja005701a.

(11) Fery-Forgues, S.; Le Bris, M. T.; Guette, J. P.; Valeur, B. Ion-Responsive Fluorescent Compounds. 1. Effect of Cation Binding on Photophysical Properties of Benzoxazinone Derivative Linked to Monoaza-15-Crown-5. J. Phys. Chem. 1988, 92 (22), 6233-6237. https://doi.org/10.1021/j100333a013.

(12) Saleh, N. Luminescent Sensor for $\mathrm{Cd} 2+, \mathrm{Hg} 2+$ and $\mathrm{Ag}+$ in Water Based on a Sulphur- 
Containing Receptor: Quantitative Binding-Softness Relationship. Luminescence 2009, 24 (1), 30-34. https://doi.org/10.1002/bio.1058.

(13) Darbha, G. K.; Ray, A.; Ray, P. C. Gold Nanoparticle-Based Miniaturized Nanomaterial Surface Energy Transfer Probe for Rapid and Ultrasensitive Detection of Mercury in Soil, Water, and Fish. ACS Nano 2007, 1 (3), 208-214. https://doi.org/10.1021/nn7001954.

(14) Keri, R. S.; Hiremathad, A.; Budagumpi, S.; Nagaraja, B. M. Comprehensive Review in Current Developments of Benzimidazole-Based Medicinal Chemistry. Chem. Biol. Drug Des. 2015, 86 (1), 19-65. https://doi.org/10.1111/cbdd.12462.

(15) Ali, E. M.; Zheng, Y.; Yu, H. H.; Ying, J. Y. Ultrasensitive Pb2+ Detection by Glutathione-Capped Quantum Dots. Anal. Chem. 2007, 79 (24), 9452-9458. https://doi.org/10.1021/ac071074x.

(16) Ding, C.; Zhu, A.; Tian, Y. Functional Surface Engineering of C-Dots for Fluorescent Biosensing and in Vivo Bioimaging. Acc. Chem. Res. 2014, 47 (1), 20-30. https://doi.org/10.1021/ar400023s.

(17) Kim, T. H.; Raiz, A.; Unni, A. D.; Murhekar, S.; Donose, B. C.; Floetenmeyer, M.; Cock, I. E.; Brown, C. L. Combating Antibiotic-Resistant Gram-Negative Bacteria Strains with Tetracycline-Conjugated Carbon Nanoparticles. Adv. Biosyst. 2020. https://doi.org/10.1002/adbi.202000074.

(18) Kim, T. H.; White, A. R.; Sirdaarta, J. P.; Ji, W.; Cock, I. E.; St. John, J.; Boyd, S. E.; Brown, C. L.; Li, Q. Yellow-Emitting Carbon Nanodots and Their Flexible and Transparent Films for White LEDs. ACS Appl. Mater. Interfaces 2016, 8 (48), 33102 33111. https://doi.org/10.1021/acsami.6b12113.

(19) Zhang, L.; Wei, H.; Gao, F.; Su, Y.; Su, Y.; Zhang, J.; Ma, Y.; Xu, M.; Xu, M.; Li, Z.; Yang, Z. Controllable Synthesis of Fluorescent Carbon Dots and Their Detection Application as Nanoprobes. Nano-Micro Lett. 2013, 5 (4), 247-259. https://doi.org/10.5101/nml.v5i4.p247-259.

(20) Wang, F.; Pang, S. P.; Wang, L.; Li, Q.; Kreiter, M.; Liu, C. Y. One-Step Synthesis of Highly Luminescent Carbon Dots in Noncoordinating Solvents. Chem. Mater. 2010, 22 (16), 4528-4530. https://doi.org/Doi 10.1021/Cm101350u.

(21) Baker, S. N.; Baker, G. A. Luminescent Carbon Nanodots: Emergent Nanolights. Angew. Chemie Int. Ed. 2010, 49 (38), 6726-6744. https://doi.org/10.1002/anie.200906623.

(22) Sun, Y. P.; Wang, X.; Lu, F.; Cao, L.; Meziani, M. J.; Luo, P. G.; Gu, L.; Monica Veca, L. Doped Carbon Nanoparticles as a New Platform for Highly Photoluminescent Dots. $J$. Phys. Chem. C 2008, 112 (47), 18295-18298. https://doi.org/10.1021/jp8076485.

(23) Dong, Y.; Wang, R.; Li, G.; Chen, C.; Chi, Y.; Chen, G. Polyamine-Functionalized Carbon Quantum Dots as Fluorescent Probes for Selective and Sensitive Detection of Copper Ions. Anal. Chem. 2012, 84 (14), 6220-6224. https://doi.org/10.1021/ac3012126.

(24) Dong, Y.; Li, G.; Zhou, N.; Wang, R.; Chi, Y.; Chen, G. Graphene Quantum Dot as a Green and Facile Sensor for Free Chlorine in Drinking Water. Anal Chem 2012, 84 (19), 8378-8382. https://doi.org/10.1021/ac301945z.

(25) Kim, T. H.; Ho, H. W.; Brown, C. L.; Cresswell, S. L.; Li, Q. Amine-Rich Carbon 
Nanodots as a Fluorescence Probe for Methamphetamine Precursors. Anal. Methods 2015, 7 (16), 6869-6876. https://doi.org/10.1039/C5AY01715G.

(26) Fujii, S.; Tsuchida, H.; Kōmoto, M. Chemical Studies on the Reaction Products of Glucose and Ammonia. Agric. Biol. Chem. 1966, 30 (1), 73-77. https://doi.org/10.1080/00021369.1966.10858548.

(27) Amendola, V.; Fabbrizzi, L.; Mosca, L. Anion Recognition by Hydrogen Bonding: UreaBased Receptors. Chem. Soc. Rev. 2010, 39 (10), 3889. https://doi.org/10.1039/b822552b.

(28) Geddes, C. D.; Lakowicz, J. R. Metal-Enhanced Fluorescence. In Journal of Fluorescence; Springer, 2002; Vol. 12, pp 121-129. https://doi.org/10.1023/A:1016875709579.

(29) Gao, X.; Lu, Y.; Zhang, R.; He, S.; Ju, J.; Liu, M.; Li, L.; Chen, W. One-Pot Synthesis of Carbon Nanodots for Fluorescence Turn-on Detection of Ag+ Based on the Ag+-Induced Enhancement of Fluorescence. J. Mater. Chem. C 2015, 3 (10), 2302-2309. https://doi.org/10.1039/c4tc02582b.

(30) Pekel, N.; Guven, O. Investigation of Complex Formation between Poly(N-Vinyl Imidazole) and Various Metal Ions Using the Molar Ratio Method. Colloid Polym. Sci. 1999, 277 (6), 570-573. https://doi.org/10.1007/s003960050426.

(31) Yassar, a; Horowitz, G.; Valat, P.; Wintgens, V.; Hmyene, M.; Deloffre, F.; Srivastava, P.; Lang, P.; Garnier, F. Exciton Coupling Effects in the Absorption and Photoluminescence of Sexithiophene Derivatives. J. Phys. Chem. 1995, 99 (22), 91559159. https://doi.org/10.1021/j100022a031.

(32) Misra, S. K.; Srivastava, I.; Tripathi, I.; Daza, E.; Ostadhossein, F.; Pan, D. Macromolecularly "Caged" Carbon Nanoparticles for Intracellular Trafficking via Switchable Photoluminescence. J. Am. Chem. Soc. 2017, 139 (5), jacs.6b11595. https://doi.org/10.1021/jacs.6b11595.

(33) Connors, K. A. (Kenneth A. Connors , K. A . Binding Constants : The Measurement of Molecular Complex Stability, John Wiley and Sons , Inc ., 1987 , Pp . 341 . ISBN : 978-0471-83083-2 ; Wiley, 2010; Vol. 47.

(34) Kuntz, I. D.; Gasparro, F. P.; Johnston, M. D.; Taylor, R. P. Molecular Interactions and the Benesi-Hildebrand Equation. J. Am. Chem. Soc. 1968, 90 (18), 4778-4781. https://doi.org/10.1021/ja01020a004.

(35) Wang, J.; Qian, X. A Series of Polyamide Receptor Based PET Fluorescent Sensor Molecules: Positively Cooperative Hg2+ Ion Binding with High Sensitivity. Org. Lett. 2006, 8 (17), 3721-3724. https://doi.org/10.1021/o1061297u.

(36) Aryal, G. H.; Battle, C. H.; Grusenmeyer, T. A.; Zhu, M.; Jayawickramarajah, J. A Naphthalimide Derived Fluorescent Sensor for Solid-Phase Screening of Cucurbit[7]UrilGuest Interactions. Chem. Commun. 2016, 52 (11), 2307-2310. https://doi.org/10.1039/C5CC08350H.

(37) Cao, L.; Meziani, M. J.; Sahu, S.; Sun, Y. P. Photoluminescence Properties of Graphene versus Other Carbon Nanomaterials. Acc. Chem. Res. 2013, 46 (1), 171-180. https://doi.org/10.1021/ar300128j. 
(38) McFarland, S. A.; Finney, N. S. Fluorescent Signaling Based on Control of Excited State Dynamics. Biarylacetylene Fluorescent Chemosensors. J. Am. Chem. Soc. 2002, 124 (7), 1178-1179. https://doi.org/10.1021/ja017309i.

(39) Monticone, S.; Tufeu, R. Complex Nature of the UV and Visible Fluorescence of Colloidal ZnO Nanoparticles. J. Phys. Chem. B 1998, 102 (16), 2854-2862. https://doi.org/10.1021/jp973425p.

(40) An, B. K.; Kwon, S. K.; Jung, S. D.; Park, S. Y. Enhanced Emission and Its Switching in Fluorescent Organic Nanoparticles. J. Am. Chem. Soc. 2002, 124 (48), 14410-14415. https://doi.org/10.1021/ja0269082.

(41) Würthner, F. Perylene Bisimide Dyes as Versatile Building Blocks for Functional Supramolecular Architectures. Chem. Commun. (Camb). 2004, 0 (14), 1564-1579. https://doi.org/10.1039/b401630k.

(42) Henary, M. M.; Fahrni, C. J. Excited State Intramolecular Proton Transfer and Metal Ion Complexation of 2-(2'-Hydroxyphenyl)Benzazoles in Aqueous Solution. J. Phys. Chem. A 2002, 106 (21), 5210-5220. https://doi.org/10.1021/jp014634j.

(43) Ma, D. L.; Ma, V. P. Y.; Chan, D. S. H.; Leung, K. H.; He, H. Z.; Leung, C. H. Recent Advances in Luminescent Heavy Metal Complexes for Sensing. Coordination Chemistry Reviews. Elsevier December 1, 2012, pp 3087-3113. https://doi.org/10.1016/j.ccr.2012.07.005.

(44) Pugmire, R. J.; Grant, D. M. Carbon-13 Magnetic Resonance. XII. Five-Membered Nitrogen Heterocycles and Their Charged Species. J. Am. Chem. Soc. 1968, 90 (16), 4232-4238. https://doi.org/10.1021/ja01018a008.

(45) Ramachandram, B.; Samanta, A. Transition Metal Ion Induced Fluorescence Enhancement of 4- ( N , N-Dimethylethylenediamino ) -7-Nitrobenz-2-Oxa-1 , 3-Diazole. J. Phys. Chem. A 1998, 102 (52), 10579-10587. https://doi.org/10.1021/JP983106D. 